\title{
Synthesis and evaluation of antimicrobial properties of some azole derivatives
}

\author{
Mohd Imran ${ }^{1 \star}$, Abdulhakim Bawadekji ${ }^{2}$, Nawaf Alotaibi ${ }^{3}$ \\ ${ }^{1}$ Department of Pharmaceutical Chemistry, Faculty of Pharmacy, Northern Border University, PO Box 840, Rafha 91911, \\ ${ }^{2}$ Department of Biological Sciences, College of Science, Northern Border University, PO Box 1321, Arar 91431, ${ }^{3}$ Department of \\ Pharmaceutics, Faculty of Pharmacy, Northern Border University, PO Box 840, Rafha 91911, Saudi Arabia
}

*For correspondence: Email: imran_inderlok@yahoo.co.in; Tel: +966-599577945

Sent for review: 18 September 2019

Revised accepted: 25 January 2020

\begin{abstract}
Purpose: To synthesize new azole derivatives and determine their antimicrobial properties.

Methods: The reaction of the intermediates (2a-2c) with 3a-3c in acetone/potassium carbonate solution yielded 4a-4i, which were characterized using Fourier-transform infrared spectroscopy (FTIR), proton nuclear magnetic resonance $\left({ }^{1} \mathrm{H}-\mathrm{NMR}\right)$, carbon-13 nuclear magnetic resonance $\left.\left({ }^{13} \mathrm{C}-\mathrm{NMR}\right)\right)$ and mass spectrometry (MS). Compounds 4a-4i were assessed for their antibacterial and antifungal effects using the sequential dilution technique, relative to ofloxacin and ketoconazole.

Results: The spectral data for $4 a-4 i$ were consistent with the assigned structures. The MIC of compound $4 \mathrm{~h}(10 \mu \mathrm{g} / \mathrm{ml})$ was similar to that of ketoconazole against Aspergillus flavus, Penicillium citrinum, and Aspergillus niger. The MIC value of compound $4 \mathrm{~b}(10 \mathrm{\mu g} / \mathrm{ml})$ for Penicillium citrinum was comparable to that of ketoconazole while the MIC value of compound $4 d$ against Staphylococcus aureus and Escherichia coli $(20 \mathrm{\mu g} / \mathrm{ml})$ was equivalent to the corresponding MIC value for ofloxacin.

Conclusion: The synthesized compounds bearing boronic acid moiety are good antimicrobial agents. Accordingly, further investigation into the thiazole-imidazole or thiazole-triazole derivatives bearing boronic acid moiety is suggested.
\end{abstract}

Keywords: Synthesis, Imidazole, Thiazole, Triazole, Antimicrobials

\begin{abstract}
This is an Open Access article that uses a fund-ing model which does not charge readers or their institutions for access and distributed under the terms of the Creative Commons Attribution License (http://creativecommons.org/licenses/by/4.0) and the Budapest Open Access Initiative (http://www.budapestopenaccessinitiative.org/read), which permit unrestricted use, distribution, and reproduction in any medium, provided the original work is properly credited.
\end{abstract}

Tropical Journal of Pharmaceutical Research is indexed by Science Citation Index (SciSearch), Scopus, International Pharmaceutical Abstract, Chemical Abstracts, Embase, Index Copernicus, EBSCO, African Index Medicus, JournalSeek, Journal Citation Reports/Science Edition, Directory of Open Access Journals (DOAJ), African Journal Online, Bioline International, Open-J-Gate and Pharmacy Abstracts

\section{INTRODUCTION}

Recent reports on microbial resistance and emergence of new microbial diseases pose serious challenges to the affected patients, as well as the health care community [1]. This challenge is further exacerbated by inappropriate antimicrobial therapy trends [2]. Therefore, scientists are making efforts to provide novel antimicrobial agents with diverse mechanisms of action from the known chemical classes of the existing antimicrobial drugs $[3,4]$.

Azole is one of the important chemical classes of antimicrobial agents [5]. The development of azole antimicrobial agents is the focus of current research [6]. Many azole-based antimicrobial agents are already in clinical use, for example, ketoconazole, econazole, miconazole, posaconazole, fluconazole, voriconazole, and 
isavuconazole. Based on these facts [5-10], the present study was carried out to synthesize new azole derivatives, and to determine their antibacterial and antifungal properties.

\section{EXPERIMENTAL}

\section{Materials and reagents}

Gallenkamp apparatus was used to determine the melting points of the synthesized compounds. The IR spectra determination $(\mathrm{KBr}$; wave number in $\mathrm{cm}^{-1}$ ), NMR analysis (DMSO- $\mathrm{d}_{6}$; $\delta$ in $\mathrm{ppm})$, mass analysis $\left(\mathrm{M}^{+} ; \mathrm{m} / \mathrm{z}\right)$, and elemental investigation ( $\mathrm{C}, \mathrm{H}$ and $\mathrm{N}$ analysis) were performed using Shimadzu spectrophotometer, Bruker DRX-300 spectrophotometer, Jeol-JMS-D-300 spectrometer, and VARIO EI Elementer apparatus, respectively. The monitoring of reactions and assessment of purity were carried out using TLC. Compounds $\mathbf{3 a - 3 c}$ were purchased from Sigma Aldrich.

\section{Synthesis of substituted phenacyl intermediates (2a-2c)}

Compounds $\mathbf{2 a - 2 c}$ were prepared using the prior art process [11]. In general, a mixture of acetophenone $(0.1 \mathrm{~mole})$ in acetic acid $(20 \mathrm{ml})$ was stirred at $80^{\circ} \mathrm{C}$ with a solution of bromine $(0.1 \mathrm{~mole})$ in acetic acid $(25 \mathrm{ml})$. The precipitate was filtered and recrystallized from ethanol.

\section{Synthesis of 4-(2-((4-phenyl-1H-imidazole-2- yl)thio)acetyl)phenyl acetate (4a)}

A mixture of $2 \mathrm{a}(0.1 \mathrm{~mole}), 3 \mathrm{a}$ (0.1 mole) and potassium carbonate $(0.1$ mole $)$ in $30 \mathrm{~mL}$ acetone was stirred at $25{ }^{\circ} \mathrm{C}$ for $15-20 \mathrm{~h}$. The reaction mixture was dissolved in water (250-500 $\mathrm{mL}$ ), and the filtered residue was purified with ethanol.

The other imidazole derivatives $(\mathbf{4 b - 4 c )}$, thiazole derivatives (4d-4f), and triazole derivatives (4g4i) were also prepared in a similar manner.

\section{Determination of antimicrobial activity}

The sequential dilution technique [12,13] was used for determination of antimicrobial effects of the synthesized compounds. A similar procedure was described in previous publications [7-10]. Different concentrations of $\mathbf{4 a - 4 i}$, ketoconazole, and ofloxacin were prepared and their MICs were determined using agar medium and sterile dimethyl sulfoxide (DMSO). The sterile DMSO also functioned as control or blank. The microorganisms tested are indicated in Table 2.

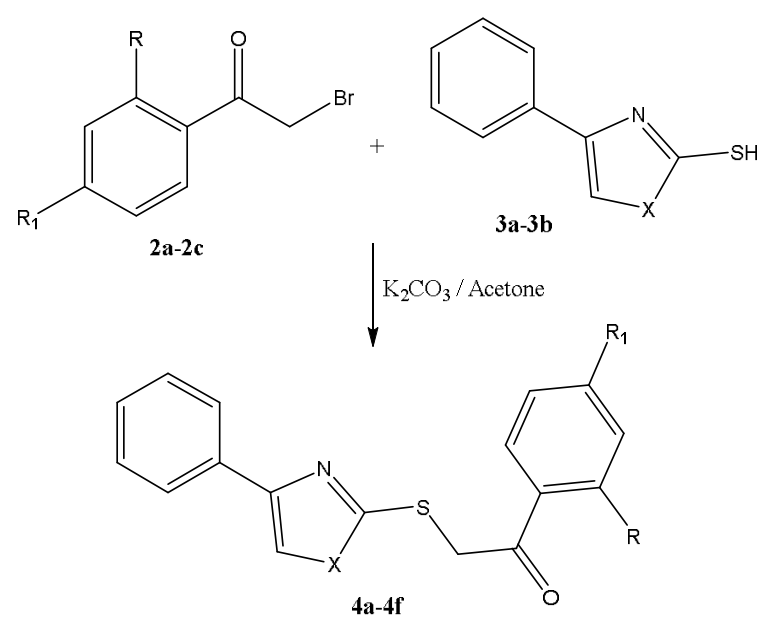

$4 \mathfrak{a}: \mathrm{X}=-\mathrm{NH}-, \mathrm{R}=-\mathrm{H}, \mathrm{R}_{1}=-\mathrm{OCOCH}_{3} ; \mathbf{4 b}: \mathrm{X}=-\mathrm{NH}-, \mathrm{R}=-\mathrm{H}, \mathrm{R}_{1}=-\mathrm{B}(\mathrm{OH})_{2}$ 4c: $\mathrm{X}=-\mathrm{NH}-, \mathrm{R}=-\mathrm{Br}, \mathrm{R}_{1}=-\mathrm{H} ; \mathbf{4 d}: \mathrm{X}=-\mathrm{S}-, \mathrm{R}=-\mathrm{H}, \mathrm{R}_{1}=-\mathrm{OCOCH}_{3}$ $4 \mathrm{~b}: \mathrm{X}=-\mathrm{S}-, \mathrm{R}=-\mathrm{H}, \mathrm{R}_{1}=-\mathrm{B}(\mathrm{OH})_{2} ; 4 \mathbf{c}: \mathrm{X}=-\mathrm{S}-, \mathrm{R}=-\mathrm{Br}, \mathrm{R}_{1}=-\mathrm{H}$;

Figure 1: Synthesis of compounds $\mathbf{4 a - 4 f}$

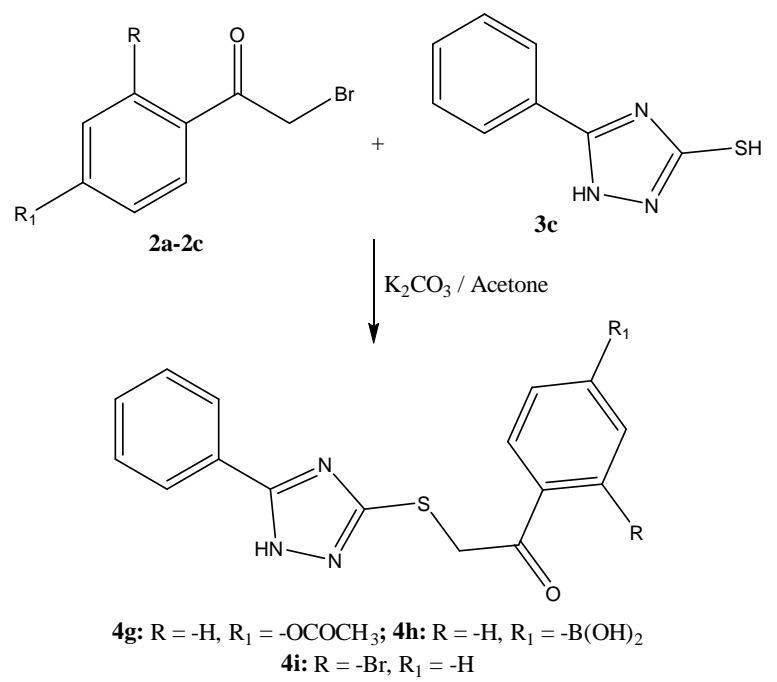

Figure 2: Synthesis of compounds $\mathbf{4 g - 4 i}$

\section{Statistical analysis}

The data are expressed as mean \pm standard error mean (S.E.M., $\mathrm{n}=3$ ). Statistical analysis was done using SPSS-software (version 20). Statistical significance was assumed at $p<0.05$.

\section{RESULTS}

Figure 1 and Figure 2 depict the synthesis of compounds $\mathbf{4 a - 4 f}$ and $\mathbf{4 g}-\mathbf{4 i}$, respectively. The reaction of $2 \mathbf{a}-\mathbf{2} \mathbf{c}$ with $\mathbf{3 a - 3} \mathbf{c}$ in acetone $/ \mathrm{K}_{2} \mathrm{CO}_{3}$ yielde $\mathbf{4 a}-\mathbf{4 i}$, which were characterized through spectral analysis.

Table 1 and Table 2 display the spectral data of compounds $4 \mathbf{a}-\mathbf{4 i}$. The IR spectra of the different compounds in $\mathbf{4 a - 4} \mathbf{i}$ series exhibited characteristic IR bands. These bands included 
characteristics peaks for $-\mathrm{OH}$ groups of $\mathbf{4 b}, \mathbf{4 e}$, and $\mathbf{4 h}$, starting from 3350 to $3360 \mathrm{~cm}^{-1}$; $-\mathrm{NH}$ groups of $\mathbf{4 a - 4 c}$ and $\mathbf{4 g - 4 i}$ from 3120 to $3125 \mathrm{~cm}^{-}$ ; and $\mathrm{C}=\mathrm{O}$ groups of the acetoxy groups of $\mathbf{4 a}$, $\mathbf{4 d}$, and $\mathbf{4 g}$, from 1730 to $1735 \mathrm{~cm}^{-1}$. Compounds 4a-4i also exhibited peaks for $\mathrm{C}=\mathrm{O}$ group from 1690 to 1695 ; $\mathrm{C}=\mathrm{N}$ group from 1640 to 1645 ; and $\mathrm{C}=\mathrm{C}$ assemblage from 1595 to $1600 \mathrm{~cm}^{-1}$.

The $1 \mathrm{H}$ NMR spectra of $\mathbf{4 a - 4} \mathbf{i}$ showed aromatic hydrogens as multiplets at $\delta 7.40-7.85$, and the methylene protons $\left(-\mathrm{CH}_{2}-\right)$ as singlets at $\delta$ 4.604.63. Compounds $\mathbf{4 a}, \mathbf{4 d}$ and $\mathbf{4 g}$ exhibited singlets at $\delta 2.12-2.15$ for the methyl group. Compounds $\mathbf{4 a - 4 c}$ and $\mathbf{4 g}-\mathbf{4} \mathbf{i}$ showed a singlet for the $-\mathrm{NH}$ - group at $\delta 11.94-1199$. The boronic acid derivatives $\mathbf{4 b}, \mathbf{4 e}$ and $\mathbf{4 h}$ displayed singlet peaks for $-\mathrm{OH}$ group at $\delta 4.11-4.15[11]$. The ${ }^{13} \mathrm{C}$ NMR spectra of $\mathbf{4 a - 4 i}$ revealed characteristic peaks of carbonyl carbon (- $\left.\mathrm{CH}_{2}-\mathrm{CO}-\right)$ at $\delta 195.0$ 195.1 and methylene carbon $\left(-\mathrm{CH}_{2}-\mathrm{CO}-\right)$ at $\delta$ 39.1-38.4. Compounds $\mathbf{4 a}, \mathbf{4 d}$ and $\mathbf{4} \mathbf{g}$ exhibited additional peaks of methyl carbon $\left(-\mathrm{CH}_{3}\right)$ at $\delta$ 21.2, and another carbonyl carbon (-CO- of acetoxy) at $\delta 168.1$ - 168.0. Other peaks appeared in the aromatic regions of the ${ }^{13} \mathrm{C}$ NMR spectra. The mass analysis $(\mathrm{m} / \mathrm{z})$ and the elemental ( $\mathrm{C}, \mathrm{H}$ and $\mathrm{N}$ ) analysis data of $\mathbf{4 a - 4 i}$ were also in agreement with the allocated structures.

Table 3 shows the antimicrobial activities of $4 a-$ 4i, wherein the MIC values are expressed in $\mu \mathrm{g} / \mathrm{ml}$. For purpose of comparison, the MIC values of ketoconazole and ofloxacin were considered as $100 \%$. The data revealed that compound $\mathbf{4 h}$ and ketoconazole had the same MIC value $(10 \mu \mathrm{g} / \mathrm{mL})$ against Aspergillus niger, Aspergillus flavus, and Penicillium citrinum, while compound $\mathbf{4 b}$ and ketoconazole had similar MIC (10 $\mu \mathrm{g} / \mathrm{mL}$ ) against Penicillium citrinum. Compound $\mathbf{4 d}$ and ofloxacin had equivalent MIC value $(20 \mu \mathrm{g} / \mathrm{mL})$ against Staphylococcus aureus and Escherichia coli. It was also obvious that compounds $\mathbf{4 d - 4 f}$ displayed very good antimicrobial activities (80 - $100 \%)$ against Staphylococcus aureus, Escherichia coli, Bacillus subtilis, and Pseudomonas aeruginosa, relative to ofloxacin, whereas compounds $\mathbf{4 a - 4 c}$ and $\mathbf{4 g -}$ 4i were moderately active.

Table 1a: Spectral data for $4 a-4 e$

\begin{tabular}{|c|c|c|c|c|c|}
\hline $\begin{array}{l}\text { Compound } \\
\text { (molecular } \\
\text { formula) } \\
\text { (melting point) }\end{array}$ & $\begin{array}{c}\text { IR } \\
\text { (Wave } \\
\text { number in } \\
\text { cm-) } \\
\end{array}$ & $\begin{array}{l}{ }^{1} \mathrm{H} \mathrm{NMR} \\
(\delta \text { in ppm, } \\
\text { DMSO-d }\end{array}$ & $\begin{array}{c}{ }^{13} \mathrm{C} \text { NMR } \\
\left(\delta \text { in ppm, DMSO-d }{ }_{6}\right)\end{array}$ & $\begin{array}{c}\text { Mass } \\
\left(\mathrm{m} / \mathrm{z} ; \mathrm{M}^{+}\right)\end{array}$ & $\begin{array}{l}\text { C, H, N Anal., } \\
\text { \{Found } \\
\text { (Calculated)\} }\end{array}$ \\
\hline $\begin{array}{l}\text { 4a } \\
\left(\mathrm{C}_{19} \mathrm{H}_{16} \mathrm{~N}_{2} \mathrm{O}_{3} \mathrm{~S}\right) \\
\left(178-180^{\circ} \mathrm{C}\right)\end{array}$ & $\begin{array}{c}3120, \\
1730, \\
1690, \\
1640, \text { and } \\
1595 \\
3350\end{array}$ & $\begin{array}{c}2.12(\mathrm{~s}, 3 \mathrm{H}) \\
4.62(\mathrm{~s}, 2 \mathrm{H}) \\
7.40-7.85(\mathrm{~m}, \\
10 \mathrm{H}), 11.95 \\
\quad(\mathrm{~s}, 1 \mathrm{H}) \\
4.11(\mathrm{~s}, 2 \mathrm{H})\end{array}$ & $\begin{array}{c}21.2\left(-\mathrm{CH}_{3}\right), 39.1\left(-\mathrm{CH}_{2-}\right) \text {, } \\
120.8,122.4(2 \mathrm{C}), 128.4 \\
(2 \mathrm{C}), 129.6,130.1(4 \mathrm{C}), \\
133.1,134.0,141.0, \\
141.6,156.6,168.1(- \\
\text { CO- of acetoxy), } 195.0(- \\
\text { CO-) } \\
39.1\left(-\mathrm{CH}_{2-}\right), 113.0\end{array}$ & 352 & $\begin{array}{c}\mathrm{C}, 64.75(64.76) ; \\
\mathrm{H}, 4.55(4.58) ; \mathrm{N}, \\
7.90(7.95)\end{array}$ \\
\hline $\begin{array}{l}\text { 4b } \\
\left(\mathrm{C}_{17} \mathrm{H}_{15} \mathrm{BN}_{2} \mathrm{O}_{3} \mathrm{~S}\right) \\
\left(144-146^{\circ} \mathrm{C}\right)\end{array}$ & $\begin{array}{c}3350, \\
3125, \\
1695, \\
1645,1600\end{array}$ & $\begin{array}{c}4.11(\mathrm{~s}, 2 \mathrm{H}), \\
4.60(\mathrm{~s}, 2 \mathrm{H}), \\
7.41-7.84(\mathrm{~m}, \\
10 \mathrm{H}), 11.98 \\
(\mathrm{~s}, 1 \mathrm{H}) \\
4.63(\mathrm{~s}, 2 \mathrm{H}),\end{array}$ & $\begin{array}{c}39.1\left(-\mathrm{CH}_{2-}^{-}\right), 113.0, \\
120.8,128.4(2 \mathrm{C}), 129.6 \\
(3 \mathrm{C}), 130.1(2 \mathrm{C}), 134.0, \\
134.2(2 \mathrm{C}), 136.3,141.0 \\
\text { 141.6, 195.1 (-CO-). } \\
38.4\left(-\mathrm{CH}_{2^{-}}\right), 120.8,\end{array}$ & 338 & $\begin{array}{c}\mathrm{C}, 60.35(60.38) ; \\
\mathrm{H}, 4.45(4.47) ; \mathrm{N}, \\
\quad 8.25(8.28)\end{array}$ \\
\hline $\begin{array}{l}\mathbf{4 c} \\
\left(\mathrm{C}_{17} \mathrm{H}_{13} \mathrm{BrN}_{2} \mathrm{OS}\right) \\
\left(161-163^{\circ} \mathrm{C}\right)\end{array}$ & $\begin{array}{c}3122, \\
1692, \\
1640,1600\end{array}$ & $\begin{array}{c}7.42-7.85(\mathrm{~m}, \\
10 \mathrm{H}), 11.99 \\
(\mathrm{~s}, 1 \mathrm{H})\end{array}$ & $\begin{array}{c}122.1,128.2,128.4(3 \mathrm{C}) \\
\text { 129.6, } 130.1(2 \mathrm{C}), 133.1 \\
\text { 133.5, } 134.0,141.0 \\
\text { 141.2, 141.6, } 195.1(- \\
\text { CO-). }\end{array}$ & $\begin{array}{c}371\left(\mathrm{M}^{+}\right) \\
\& 373 \\
\left(\mathrm{M}^{+}+2\right)\end{array}$ & $\begin{array}{c}\mathrm{C}, 54.65(54.70) ; \\
\mathrm{H}, 3.50(3.51) ; \mathrm{N}, \\
7.45(7.51)\end{array}$ \\
\hline $\begin{array}{l}\text { 4d } \\
\left(\mathrm{C}_{19} \mathrm{H}_{15} \mathrm{NO}_{3} \mathrm{~S}_{2}\right) \\
\left(171-173^{\circ} \mathrm{C}\right)\end{array}$ & $\begin{array}{c}1732, \\
1695 \\
1641,1598\end{array}$ & $\begin{array}{c}2.15(\mathrm{~s}, 3 \mathrm{H}), \\
4.61(\mathrm{~s}, 2 \mathrm{H}), \\
7.40-7.83(\mathrm{~m}, \\
10 \mathrm{H})\end{array}$ & $\begin{array}{c}21.2\left(-\mathrm{CH}_{3}\right), 39.1\left(-\mathrm{CH}_{2-}\right), \\
113.1,122.4(2 \mathrm{C}), 128.4 \\
(2 \mathrm{C}), 129.6,130.1(4 \mathrm{C}), \\
133.1,134.0,155.8, \\
\text { 156.6, 167.6, 168.0 (- } \\
\text { CO- of acetoxy), } 195.0(- \\
\text { CO-). }\end{array}$ & 369 & $\begin{array}{c}\mathrm{C}, 61.75 \text { (61.77); } \\
\mathrm{H}, 4.04 \text { (4.09); N, } \\
3.77 \text { (3.79) }\end{array}$ \\
\hline $\begin{array}{l}4 \mathrm{e} \\
\left(\mathrm{C}_{17} \mathrm{H}_{14} \mathrm{BNO}_{3} \mathrm{~S}_{2}\right) \\
\left(151-153^{\circ} \mathrm{C}\right)\end{array}$ & $\begin{array}{c}3360, \\
1695, \\
1645,1600\end{array}$ & $\begin{array}{c}4.15(\mathrm{~s}, 2 \mathrm{H}), \\
4.62(\mathrm{~s}, 2 \mathrm{H}), \\
7.44-7.85(\mathrm{~m}, \\
10 \mathrm{H})\end{array}$ & $\begin{array}{c}39.1\left(-\mathrm{CH}_{2-}^{-}\right), 113.0 \\
113.3,128.4(2 \mathrm{C}), 129.6 \\
(3 \mathrm{C}), 130.1(2 \mathrm{C}), 134.0 \\
134.2(2 \mathrm{C}), 136.3,155.8 \\
167.6,195.1(-\mathrm{CO}-) .\end{array}$ & 355 & $\begin{array}{c}\text { C, } 57.45 \text { (57.48); } \\
\text { H, } 3.95(3.97) ; N \text {, } \\
3.90(3.94)\end{array}$ \\
\hline
\end{tabular}


Table 2: Spectral data for $\mathbf{4 f - 4 i}$

\begin{tabular}{|c|c|c|c|c|c|}
\hline $\begin{array}{l}\text { Compound } \\
\text { (molecular } \\
\text { formula) } \\
\text { (melting point) }\end{array}$ & $\begin{array}{c}\text { IR } \\
\text { (Wave } \\
\text { number in } \\
\text { cm-) }\end{array}$ & $\begin{array}{l}{ }^{1} \mathrm{H} \text { NMR } \\
(\delta \text { in ppm, } \\
\left.\text { DMSO-d })_{6}\right)\end{array}$ & $\begin{array}{c}{ }^{13} \mathrm{C} \text { NMR } \\
\left(\delta \text { in ppm, DMSO- } d_{6}\right)\end{array}$ & $\begin{array}{c}\text { Mass } \\
\left(\mathrm{m} / \mathbf{z} ; \mathbf{M}^{+}\right)\end{array}$ & $\begin{array}{l}\text { C, H, N Anal., } \\
\text { \{Found } \\
\text { (Calculated)\} }\end{array}$ \\
\hline $\begin{array}{l}\mathbf{4 f} \\
\left(\mathrm{C}_{17} \mathrm{H}_{12} \mathrm{BrNOS}_{2}\right) \\
\left(167-169^{\circ} \mathrm{C}\right)\end{array}$ & $\begin{array}{c}1690 \\
1640,1595\end{array}$ & $\begin{array}{c}4.62(\mathrm{~s}, 2 \mathrm{H}) \\
7.41-7.83(\mathrm{~m}, \\
10 \mathrm{H}) \\
2.14(\mathrm{~s}, 3 \mathrm{H})\end{array}$ & $\begin{array}{c}\text { 38.4 }\left(-\mathrm{CH}_{2^{-}}\right), 113.1, \\
122.1,128.2,128.4(3 \mathrm{C}), \\
129.6,130.1(2 \mathrm{C}), 133.1 \\
\text { 133.5, } 134.0,141.2, \\
155.8,167.6,195.1(- \\
\mathrm{CO}-) . \\
21.2\left(-\mathrm{CH}_{3}\right), 39.1\left(-\mathrm{CH}_{2^{-}}\right),\end{array}$ & $\begin{array}{c}388\left(\mathrm{M}^{+}\right) \\
\& 390 \\
\left(\mathrm{M}^{+}+2\right)\end{array}$ & $\begin{array}{l}\mathrm{C}, 52.25(52.31) ; \\
\mathrm{H}, 3.05(3.10) ; \mathrm{N}, \\
3.55(3.59)\end{array}$ \\
\hline $\begin{array}{l}\mathbf{4 g} \\
\left(\mathrm{C}_{18} \mathrm{H}_{15} \mathrm{~N}_{3} \mathrm{O}_{3} \mathrm{~S}\right) \\
\left(131-133^{\circ} \mathrm{C}\right)\end{array}$ & $\begin{array}{c}3125, \\
1735, \\
1695, \\
1645,1600\end{array}$ & $\begin{array}{c}4.62(\mathrm{~s}, 2 \mathrm{H}), \\
7.41-7.83(\mathrm{~m}, \\
9 \mathrm{H}), \begin{array}{c}11.95(\mathrm{~s}, \\
1 \mathrm{H})\end{array}\end{array}$ & $\begin{array}{c}122.4(2 \mathrm{C}), 128.4(2 \mathrm{C}) \\
130.1(4 \mathrm{C}), 132.0,133.1 \\
\text { 133.4, 156.6, 159.8, } \\
\text { 161.4, 168.0 (-CO- of } \\
\text { acetoxy), 195.1 (-CO-). }\end{array}$ & 353 & $\begin{array}{c}\mathrm{C}, 61.13(61.18) ; \\
\mathrm{H}, 4.23(4.28) ; \mathrm{N}, \\
\quad 11.83(11.89)\end{array}$ \\
\hline $\begin{array}{l}\text { 4h } \\
\left(\mathrm{C}_{16} \mathrm{H}_{14} \mathrm{BN}_{3} \mathrm{O}_{3} \mathrm{~S}\right) \\
\left(140-142^{\circ} \mathrm{C}\right)\end{array}$ & $\begin{array}{c}3357, \\
3125, \\
1695 \\
1645,1595\end{array}$ & $\begin{array}{l}4.12(\mathrm{~s}, 2 \mathrm{H}) \\
4.61(\mathrm{~s}, 2 \mathrm{H}) \\
7.41-7.84(\mathrm{~m}, \\
9 \mathrm{H}), 11.95(\mathrm{~s}, \\
1 \mathrm{H})\end{array}$ & $\begin{array}{c}39.1\left(-\mathrm{CH}_{2}-\right), 113.0 \\
128.4(2 \mathrm{C}), 129.7(2 \mathrm{C}), \\
130.1(2 \mathrm{C}), 132.0,133.4 \\
134.2(2 \mathrm{C}), 136.3,159.5 \\
161.4,195.0(-\mathrm{CO}-)\end{array}$ & 339 & $\begin{array}{c}\mathrm{C}, 56.61(56.66) ; \\
\mathrm{H}, 4.12(4.16) ; \mathrm{N}, \\
\quad 12.26(12.39)\end{array}$ \\
\hline $\begin{array}{l}\mathbf{4 i} \\
\left(\mathrm{C}_{16} \mathrm{H}_{12} \mathrm{BrN}_{3} \mathrm{OS}\right) \\
\left(173-175^{\circ} \mathrm{C}\right)\end{array}$ & $\begin{array}{c}3120, \\
1690, \\
1643,1600\end{array}$ & $\begin{array}{c}4.60(\mathrm{~s}, 2 \mathrm{H}), \\
7.40-7.85(\mathrm{~m}, \\
9 \mathrm{H}), \begin{array}{c}11.94(\mathrm{~s}, \\
1 \mathrm{H})\end{array}\end{array}$ & $\begin{array}{c}38.4\left(-\mathrm{CH}_{2-}\right), 122.1 \\
128.2,128.4(3 \mathrm{C}), 130.1 \\
(2 \mathrm{C}), 132.0,133.1,133.4 \\
(2 \mathrm{C}), 141.2,159.5 \\
161.4,195.1 \text { (-CO-). }\end{array}$ & $\begin{array}{c}372\left(\mathrm{M}^{+}\right) \\
\& 374 \\
\left(\mathrm{M}^{+}+2\right)\end{array}$ & $\begin{array}{c}\mathrm{C}, 51.31(51.35) \\
\mathrm{H}, 3.20(3.23) ; \mathrm{N}, \\
\quad 11.18(11.23)\end{array}$ \\
\hline
\end{tabular}

Moreover, compounds $\mathbf{4 d - 4 f}$ were least active against Candida albicans, Aspergillus flavus, Penicillium citrinum and Aspergillus niger, whereas compounds $\mathbf{4 a - 4 c}$ and $\mathbf{4 g - 4 i}$ displayed moderate-to-equivalent antifungal activities, relative to ketoconazole.

\section{DISCUSSION}

The structure-activity analysis of compounds 4a$4 \mathbf{i}$ revealed that compounds of the triazole series $(\mathbf{4 g}-\mathbf{4 i})$ were more potent antifungal agents than those of the imidazole series $(\mathbf{4 a - 4 c )}$ ) and the thiazole series (4d-4f). This is in line with a previous report [14] which indicated that the azole ring must contain at least two nitrogen atoms for enhanced effects of the azole antifungal agents because the nitrogen atom in position 3 of imidazole ring and triazole ring is essential for the binding of fungal enzymes. This also accounts for the fact that compounds $\mathbf{4 d - 4 f}$ which lack nitrogen at position 3 , were the least potent of the thiazole compounds against the tested fungal strains. It was also obvious that the thiazole derivatives (4d-4f) were more potent antibacterial agents than the imidazole (4a-4c) and triazole $(\mathbf{4 g}-\mathbf{4 i})$ compounds.

It has been established that benzothiazole-based compounds have promising antibacterial effects [15]. There is a possibility that the 4- phenylthiazole moiety of compounds $\mathbf{4 d - 4 f}$ might be working like benzothiazole-based compounds [15]. In addition, the most potent antifungal compound (4h), and another promising antifungal agent (4b) also contain boronic acid moieties, which are also supposed to increase their antifungal potencies [16]. No effect on antimicrobial effect was seen with compounds containing bromine atom. However, the fluorinated derivatives of $\mathbf{4 a - 4 i}$ must be assessed for their antimicrobial potential, since many fluorinated azole antifungal agents [16,17], and fluorinated antibacterial agents [18] are in clinical use. Finally, the presence of the acetoxy group in compounds $\mathbf{4 a}, \mathbf{4 d}$ and $\mathbf{4 g}$ was not supposed to provide any additional antibacterial benefits.

\section{CONCLUSION}

Compounds $\mathbf{4 h}, \mathbf{4 b}$ and $\mathbf{4 d}$ have been identified as promising antimicrobial agents. However, they need to be evaluated against other bacteria and fungi. It has been established that the presence of a fused thiazole-imidazole or thiazole-triazole ring system in the $\mathbf{4 a - 4} \mathbf{i}$ types of compounds, along with a boronic acid moiety, may provide better broad-spectrum antimicrobial agents. The incorporation of fluorine in the structure may also provide beneficial outcomes. 
Table 3: Antimicrobial effects of compounds 4a-4i

\begin{tabular}{|c|c|c|c|c|c|c|c|c|}
\hline \multirow[b]{2}{*}{ Compound } & \multicolumn{8}{|c|}{$\mathrm{MIC}^{a}$ in $\mu \mathrm{g} / \mathrm{ml}$ (\% inhibition, relative to standard) } \\
\hline & $\begin{array}{l}\text { Candida } \\
\text { albicans }\end{array}$ & $\begin{array}{l}\text { Aspergillus } \\
\text { niger }\end{array}$ & $\begin{array}{l}\text { Aspergillus } \\
\text { flavus }\end{array}$ & $\begin{array}{l}\text { Penicillium } \\
\text { citrinum }\end{array}$ & $\begin{array}{l}\text { Staphylococcus } \\
\text { aureus }\end{array}$ & Bacillus subtilis & $\begin{array}{l}\text { Escherichia } \\
\text { coli }\end{array}$ & $\begin{array}{l}\text { Pseudomonas } \\
\text { aeruginosa }\end{array}$ \\
\hline $4 \mathbf{a}$ & $20(50 \%)$ & $25(40 \%)$ & $25(40 \%)$ & $20(50 \%)$ & $30(66.66 \%)$ & $40(50 \%)$ & $30(66.66 \%)$ & $30(66.66 \%)$ \\
\hline 4b & $12.5(80 \%)$ & $12.5(80 \%)$ & $15(66.66 \%)$ & $10(100 \%)$ & $30(66.66 \%)$ & $30(66.66 \%)$ & $30(66.66 \%)$ & $30(66.66 \%)$ \\
\hline 4c & $15(66.66 \%)$ & $15(66.66 \%)$ & $15(66.66 \%)$ & $15(66.66 \%)$ & $25(80 \%)$ & $25(80 \%)$ & $30(66.66 \%)$ & $25(80 \%)$ \\
\hline 4d & $40(25 \%)$ & $40(25 \%)$ & $40(25 \%)$ & $40(25 \%)$ & $20(100 \%)$ & $25(80 \%)$ & $20(100 \%)$ & $22.5(88.88 \%)$ \\
\hline $4 \mathrm{e}$ & $30(33.33 \%)$ & $30(33.33 \%)$ & $30(33.33 \%)$ & $30(33.33 \%)$ & $25(80 \%)$ & $25(80 \%)$ & $25(80 \%)$ & $25(80 \%)$ \\
\hline $4 f$ & $30(33.33 \%)$ & $30(33.33 \%)$ & $30(33.33 \%)$ & $30(33.33 \%)$ & $25(80 \%)$ & $25(80 \%)$ & $25(80 \%)$ & $25(80 \%)$ \\
\hline $4 \mathrm{~g}$ & $25(40 \%)$ & $25(40 \%)$ & $30(33.33 \%)$ & $25(40 \%)$ & $50(40 \%)$ & $40(50 \%)$ & $40(50 \%)$ & $50(40 \%)$ \\
\hline $4 \mathrm{~h}$ & $12.5(80 \%)$ & $10(100 \%)$ & $10(100 \%)$ & $10(100 \%)$ & $40(50 \%)$ & $40(50 \%)$ & $40(50 \%)$ & $40(50 \%)$ \\
\hline $4 i$ & $15(66.66 \%)$ & $15(66.66 \%)$ & $15(66.66 \%)$ & $15(66.66 \%)$ & $30(66.66 \%)$ & $50(0 \%)$ & $40(50 \%)$ & $30(66.66 \%)$ \\
\hline Ofloxacin & - & - & - & - & $20(100 \%)$ & $20(100 \%)$ & $20(100 \%)$ & $20(100 \%)$ \\
\hline Ketoconazole & $10(100 \%)$ & $10(100 \%)$ & $10(100 \%)$ & $10(100 \%)$ & - & - & - & - \\
\hline Control & 0.0 & 0.0 & 0.0 & 0.0 & 0.0 & 0.0 & 0.0 & 0.0 \\
\hline
\end{tabular}

$P<0.05$, compared to control 


\section{DECLARATIONS}

\section{Acknowledgement}

The authors gratefully acknowledge the support for this research study via grant no. 7659-PHM2018-3-9-F from the Deanship of Scientific Research at Northern Border University, Arar, Saudi Arabia.

\section{Conflict of interest}

No conflict of interest is associated with this work.

\section{Contribution of authors}

We declare that this work was done by the authors named in this article and all liabilities pertaining to claims relating to the content of this article will be borne by the authors.

\section{Open Access}

This is an Open Access article that uses a funding model which does not charge readers or their institutions for access and distributed under the terms of the Creative Commons Attribution License (http://creativecommons.org/licenses/by/ 4.0) and the Budapest Open Access Initiative (http://www.budapestopenaccessinitiative.org/rea d), which permit unrestricted use, distribution, and reproduction in any medium, provided the original work is properly credited.

\section{REFERENCES}

1. Semret M, Haraoui LP. Antimicrobial Resistance in the Tropics. Infect Dis Clin North Am 2019; 33(1): 231-245.

2. Paphitou NI. Antimicrobial resistance: action to combat the rising microbial challenges. Int $J$ Antimicrob Agents 2013; 42 Suppl: S25-28.

3. Uchil RR, Kohli GS, Katekhaye VM, Swami OC. Strategies to combat antimicrobial resistance. J Clin Diagn Res 2014; 8(7): ME01-04.

4. Bilal M, Rasheed T, lqbal HMN, Hu H, Wang W, Zhang $X$. Macromolecular agents with antimicrobial potentialities: $A$ drive to combat antimicrobial resistance. Int J Biol Macromol 2017; 103: 554-574.

5. Peng XM, Cai GX, Zhou CH. Recent developments in azole compounds as antibacterial and antifungal agents. Curr Top Med Chem 2013; 13(16): 1963-2010.
6. Zhang HZ, Gan LL, Wang H, Zhou CH. New Progress in Azole Compounds as Antimicrobial Agents. Mini Rev Med Chem 2017; 17(2): 122-166.

7. Imran M, Bakht MA, Samad A, Abida. Synthesis of some quinoline-pyrazoline-based naphthalenyl thiazole derivatives and their evaluation as potential antimicrobial agents. Trop J Pharm Res 2017; 16(5): 1147-1155.

8. Imran M, Abida, Khan SA. Synthesis and antimicrobial activity of some 2-amino-4-(7-substituted/unsubstituted coumarin-3-yl)-6-(chlorosubstitutedphenyl) pyrimidines. Trop J Pharm Res 2015; 14(7): 1265-1272.

9. Imran M, Abida, Alsalman AJ. Synthesis and evaluation of antimicrobial activity of some 2-morpholinomethylamino-4-(7-unsubstituted/substituted coumarin3-yl)-6-chlorosubstitutedphenyl pyrimidines. Trop J Pharm Res 2016; 15(2): 393-404.

10. Ansari MI, Khan SA. Synthesis and antimicrobial activity of some novel quinoline-pyrazoline-based coumarinyl thiazole derivatives. Med Chem Res 2017; 26(7): 1481 1496.

11. Jiang $Q$, Sheng W, Guo C. Synthesis of phenacyl bromides via K2S2O8-mediated tandem hydroxybromination and oxidation of styrenes in water. Green Chem 2013; 15: 2175-2179.

12. Barry AL. Procedures and theoretical considerations for testing antimicrobial agents in agar media. In: Lorian $\mathrm{V}$, Ed. Antibiotics in Laboratory Medicine, 3rd edn. Baltimore, Williams \& Wilkins; 1991; pp 1-16. 24.

13. Varma RS, Khan ZK, Singh AP. Eds. Antifungal agents: past, present, future prospects. Lucknow: National Academy of Chemistry and Biology, India, 1998; pp 55128.

14. Kelemen H, Orgovan G, Szekely-Szentmiklosi B. The pharmaceutical chemistry of azole antifungals. Acta Pharm Hung 2016; 86(3): 85-98.

15. Gjorgjieva $M$, Tomašič $T$, Kikelj $D$, Mašič $L P$. Benzothiazole-based Compounds in Antibacterial Drug Discovery. Curr Med Chem 2018; 25(38): 5218-5236.

16. Sharma $N$, Sharma $D$. An upcoming drug for onychomycosis: Tavaborole. J Pharmacol Pharmacother 2015; 6(4): 236-239.

17. Peyton LR, Gallagher S, Hashemzadeh M. Triazole antifungals: A review. Drugs of Today 2015; 51(12): 705-718.

18. Sharma $P C$, Jain $A$, Jain $S$. Fluoroquinolone antibacterials: $A$ review on chemistry, microbiology and therapeutic prospects. Acta Pol Pharm 2009; 66(6): 587604. 